\title{
Estudio Morfométrico del Foramen Parietal
}

\author{
Morphometry Study of the Parietal Foramen
}

Erika Collipal; Héctor Silva; Felipe Quintas; Cristian Martínez \& Mariano del Sol

COLLIPAL, E.; SILVA, H.; QUINTAS, F.; MARTíNEZ, C. \& DEL SOL, M. Estudio morfométrico del foramen parietal. Int. J. Morphol., 27(2):481-484, 2009.

RESUMEN: Los huesos parietales habitualmente presentan pequeños forámenes, estos en número de dos se encuentran situados próximo de la unión de la sutura sagital con la lambdoidea. Éstos forámenes permiten que venas emisarias drenen en el seno longitudinal superior a venas occipitales y, algunas veces, transmite una pequeña rama de la arteria occipital. Se efectuó un estudio anatómico en 39 calotas, en buen estado de conservación, de individuos adultos, del museo de la Unidad de Anatomía, Universidad de La Frontera, Chile. Se registraron los siguientes datos: presencia del foramen parietal, distancia desde el lambda al foramen parietal y desde el foramen parietal a la sutura sagital, diámetro del foramen parietal. El 58,9\% de las calotas presentaban cada uno de los huesos parietales un foramen; el 25,6\% presentaba un foramen en un parietal no existiendo en el hueso contralateral; y el 15,3\% no presentaba foramen parietal. La distancia promedio del lambda al foramen parietal fue de $33,25 \mathrm{~mm}$; la distancia de la sutura sagital al foramen parietal fue en promedio de 6,29mm; el promedio del diámetro máximo del foramen parietal fue de 2,65mm y el mínimo de $0,37 \mathrm{~mm}$. Estos datos anatómicos del foramen parietal aportan nuevos antecedentes para futuros estudios.

PALABRAS CLAVE: Anatomía; Hueso parietal; Foramen parietal.

\section{INTRODUCCIÓN}

Los huesos parietales habitualmente presentan los llamados forámenes parietales, estos en número de dos generalmente se encuentran situados cerca de la intersección de la sutura sagital con la sutura lambdoidea. Perfora el hueso parietal cerca de la sutura sagital unos pocos centímetros frente al lambda y transmite una pequeña vena emisaria y algunas veces una pequeña rama de la arteria occipital Berry \& Berry (1967).

Para el estudio antropológico del cráneo y cara se utilizan un gran numero de puntos precisos que sirven de referencia y permiten numerosas medidas, uno de estos puntos es el obelion, que esta situado a la altura de los dos forámenes parietales o de uno de ellos si el otro falta. En la parte del borde superior que corresponde al foramen parietal, los dentellones óseos disminuyen o desaparecen por completo, a esta zona de la sutura sagital se le denomina obelion Testut \& Latarjet (1971).

El número de forámenes parietales es variable, a veces se encuentra uno solo, pueden existir tres, dos a un lado y uno en el opuesto pudiendo existir cuatro, dos a cada lado según Testut \& Latarjet. Por otra parte, Williams et. al. (1995) señalaron que el foramen parietal puede estar ausente en uno o ambos lados, perforar el hueso próximo a la sutura sagital, cerca de $3,5 \mathrm{~cm}$ al frente del lambda. Para Hamilton (1982) aproximadamente a $3 \mathrm{~cm}$ delante del punto lambda existen de cada lado de la sutura sagital dos forámenes parietales.

Según Currarino (1976) el foramen parietal pequeño representa probablemente la persistencia más lateral y notable del foramen parietal primitivo.

Dependiendo de la población estudiada el foramen parietal puede estar presente en ambos lados entre el 43,2 y el 62,7 \% (Berry, 1975); entre 22,2 y $62 \%$ (Berry \& Berry); en un $40 \%$ (Berge \& Bergman, 2001).

En un estudio realizado por Boryslawski et al. (2002) categorizaron el diámetro del foramen parietal en 3 grupos, I: diámetro inferior a $0,5 \mathrm{~mm}$. II: entre 0,6 a $1,5 \mathrm{~mm}$. III: sobre 1,6 mm de diámetro. 
El foramen parietal, en situación normal, mide alrededor de $1 \mathrm{~mm}$ o menos de diámetro y permite que las ramas venosas occipitales externas lleguen al seno longitudinal superior y a venas emisarias provenientes del seno longitudinal a venas occipitales. Por otra parte, Tubbs et al. (2003) han señalado que los forámenes parietales gigantes que exceden los $5 \mathrm{~mm}$ de diámetro no son sinónimo del foramen parietal.

Moyano et al. (2008) señalaron que los forámenes parietales ensanchados, también llamados foramina paritallia permagna o forámenes parietales gigantes, son un defecto congénito y pueden ser identificados en el examen físico y confirmados imaginológicamente. Por otra parte, Oliva et al. (2001) señalaron que forámenes parietales gigantes corresponderían a una variante de la osificación normal. Este tipod e forámenes de acuerdo a lo señalado por Kutilek (1997) se transmiten a través de herencia autosómica dominante,

Para Currarino los forámenes parietales grandes representarían una anomalía de osificación del hueso parietal no relacionada con los forámenes parietales pequeños. La prevalencia de forámenes agrandados se encuentra en el rango de 1:15.000 o de 1:25.000.

Con el propósito de aportar mayores datos al conocimiento anatómico y morfométrico del foramen parietal, realizamos un estudio tendiente a verificar la presencia del foramen como también su posición y diámetros.

\section{MATERIAL Y MÉTODO}

El material de estudio correspondió a 39 calotas de sujetos adultos de la colección de piezas óseas del Museo de Anatomía, de la Facultad de Medicina, Universidad de La Frontera, Temuco, Chile. No fueron considerados aspectos de raza, edad, sexo. Las medidas fueron tomadas con un paquímetro digital y una guincha métrica de precisión.

Se observó la presencia del foramen parietal, midiéndose el diámetro del foramen y las distancias desde el foramen hasta el lambda y a la sutura sagital.

\section{RESULTADOS}

Constatamos la presencia del foramen parietal y determinamos los valores de centralización y dispersión de las variables estudiadas (Tablas I y II). La Fig. 1 ilustra la distancia del foramen a la sutura sagital y el tamaño del foramen parietal.
La presencia de un foramen en cada hueso parietal se presentó en 23 casos $(58,97)$; un foramen estuvo presente en 10 huesos parietales $(25,64 \%)$, de éstos 6 en el lado derecho (15,38\%) y 4 en el parietal izqquierdo (10,25\%); En 6 calotas en los huesos parietales no se visualizó foramen,

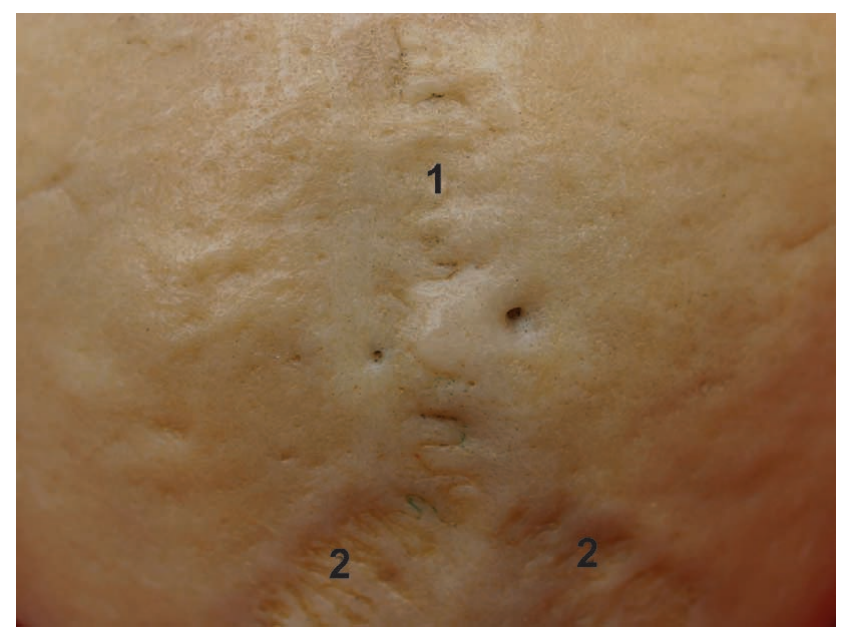

Fig. 1. Fotografía de la caalota donde se observan: 1. Sutura sagital; 2. Sutura lamboidea y dos forámenes parietales, siendo el derecho más grande que el izquierdo.

\section{DISCUSIÓN}

Como es conocido el hueso parietal se osifica a partir de dos centros primarios. Sin embargo, como indica Shaefer (1953) cuando existen defectos de osificación en estos centros, el foramen parietal es más grande de lo común.

En la cara externa de los huesos parietales y parte posterior próximo al borde superior o sagital, se encuentra el foramen parietal que da paso a una vena del seno sagital superior y, algunas veces, a una pequeña rama de la arteria occipital. Este foramen no siempre se encuentra presente como ya ha sido señalado por Williams et al.

La frecuencia del foramen parietal en las calotas examinadas mostró que en el 58,9\% el foramen parietal se encuentra presente en ambos lados. Berry \& Berry observaron la presencia del foramen parietal en varios grupos étnicos, señalaron su presencia en egipcios $(44,2 \%)$, en nigerianos $(59,2 \%)$, palestinos antiguos $(35,2 \%)$, palestinos modernos $(22,2 \%)$, hindúes $(50.0 \%)$, birmanos $(50.0 \%)$, norteamericanos (62.0\%), sudamericanos (53.0\%). Además, compararon, la incidencia del foramen parietal en relación a las diferencias por sexo, en cráneos de colección de Londres, hombres $(62,7 \%)$, mujeres $(60,4 \%)$; con mexicanos, hombres $(47,7 \%)$, mujeres $(51,6 \%)$; norteamericanos, hombres $(43,2 \%)$, mujeres $(50,0 \%)$; birmanos, hombres $(50,0 \%)$, mujeres $(50,0 \%)$. 
COLLIPAL, E.; SILVA, H.; QUINTAS, F.; MARTíNEZ, C. \& DEL SOL, M. Estudio morfométrico del foramen parietal. Int. J. Morphol., 27(2):481-484, 2009.

Tabla I. Número de forámenes parietales en 39 cráneos de sujetos adultos de la colección de piezas óseas del Museo de Anatomía, de la Facultad de Medicina, Universidad de La Frontera, Temuco, Chile.

\begin{tabular}{lcccccc}
\hline Número de forámenes & $\mathrm{n}$ & $\%$ & $\mathrm{n}$ & $\%$ & $\mathrm{n}$ & $\%$ \\
\hline Dos & 23 & 58,97 & 23 & 58,97 & 23 & 58,97 \\
Un & 10 & 25,64 & 6 & 15,38 & 4 & 10,25 \\
Ausente & 6 & 15,38 & 10 & 25,64 & 12 & 30,76 \\
\hline
\end{tabular}

Tabla II. Parámetros morfométricos de los forámenes parietales de 39 cráneos de sujetos adultos de la colección de piezas óseas del Museo de Anatomía, de la Facultad de Medicina, Universidad de La Frontera, Temuco, Chile.

\begin{tabular}{lcccc}
\hline & Promedio & Mediana & Máximo & Mínimo \\
\hline Longitud lambda-foramen parietal derecho & 32,65 & 33,0 & 41,0 & 16,0 \\
Longitud lambda-foramen parietal izquier do & 33,85 & 34,0 & 43,0 & 25,0 \\
Longitud sutura sagital-foramen parietal dererecho & 6,13 & 6,66 & 9,71 & 0,97 \\
Longitud sutura sagital-foramen parietal izquierdo & 6,45 & 6,20 & 11,21 & 1,26 \\
Diámetro del foramen parietal derecho & 1,19 & 1,05 & 2,63 & 0,31 \\
Diámetro del foramen parietal izquierdo & 1,32 & 0,98 & 2,67 & 0,44 \\
\hline
\end{tabular}

Gualdi-Russo \& Brasili (1999) determinaron que el foramen parietal estaba ausente en ambos huesos parietales de los individuos en un $54 \%$, siendo su presencia bilateral bilateral sólo en un 16,3\%. Por otro lado, Berge \& Bergman (2001) reportaron una frecuencia bilateral del foramen parietal en el $40 \%$, unilateral en el $30 \%$ y ausente en el $20 \%$, valores bastante diferentes a nuestro estudio.

Boryslawski et al. categorizaron el diámetro del foramen parietal en 3 grupos, indicando que el mayor porcentaje de forámenes estaban incluidos en el grupo III, esto es, forámenes con un diámetro mayor a 1,6mm. Comparando los datos de este estudio, el diámetro más frecuente encontrado en las calotas correspondería al grupo II, esto es entre $0,6 \mathrm{~mm}$ a $1,5 \mathrm{~mm}$ de diámetro. El foramen con mayor diámetro solo alanzó a 2,67mm, siendo el menor de $0,31 \mathrm{~mm}$, no existiendo diferencias estadísticamente significativas entre los lados derecho e izquierdo.

La localización del foramen parietal en relación al lambda en nuestro estudio fue en promedio de $3,26 \mathrm{~cm}$ en el lado derecho y a $3,38 \mathrm{~cm}$ en el lado izquierdo, similar a lo señalado por Tubbs et al., quienes lo situaron entre los rangos de 2 a $5 \mathrm{~cm}$ en cráneos de adultos.

Según Reddy et al. el hallazgo de forámenes parietales grandes, mayor a $5,0 \mathrm{~mm}$, sugiere que, probablemente, hubo un desarrollo aberrante del sistema vascular fetal y que esto podría afectar el desarrollo tanto del cráneo como del cerebro e irrigación cerebral. Por otra parte, Valente $\&$ Valente (2004) en un estudio realizado en una familia con epilepsia encontraron en la neuroimagen que las anomalías corticales y la malformación se encontraba en la región del foramen parietal magno y la presencia de este foramen incluía al miembro de la familia asintomático.

El aporte de estos datos morfométricos del foramen parietal en el hombre permite incrementar el conocimiento anatómico y sirve de base para futuros estudios anátomo clínicos.

COLLIPAL, E.; SILVA, H.; QUINTAS, F.; MARTÍNEZ, C. \& DEL SOL, M. Morphometric study of the parietal foramen. Int. J. Morphol., 27(2):481-484, 2009.

SUMMARY: The bone parietal foramina usually minor, in these two numbers are located near the junction of the sagittal suture with lambdoidea. These foramina allow emissary veins draining into the superior longitudinal sinus in the occipital veins, and sometimes sends a small branch of the occipital artery. Anatomical studies in 39 cranium, well-preserved, adult, of the Anatomy Museum of the Universidad de La Frontera, Chile was realized. Recorded the following data: presence of the parietal foramen, the distance from the lambda and the parietal foramen parietal foramen to the sagittal suture and diameter of the parietal foramen. The $58.9 \%$ of the cranium presented each of the parietal bone foramen, $25.6 \%$ had a parietal foramen in a bone in the absence of contralateral and $15.3 \%$ had no parietal foramen. The average distance from lambda to parietal foramen was $33.25 \mathrm{~mm}$, the distance from the sagittal suture to the parietal foramen averaged $6.29 \mathrm{~mm}$, the average maximum diameter of the parietal foramen was $2.65 \mathrm{~mm}$ and a minimum of $0,37 \mathrm{~mm}$. These anatomical data provide new parietal foramen of the background for future studies.

KEY WORDS: Anatomy; parietal bone, parietal foramina. 


\section{REFERENCIAS BIBLIOGRÁFICAS}

Berge, J. K \& Bergman, R. A. Variations in Size and in Symmetry of Foramina of the Human Skull. Clinical Anatomy, 14:406-413. 2001.

Berry A, C. Factors affecting the incidence of non-metrical skeletal variants. J. Anat., 120(3):519-35, 1975.

Berry, A. C \& Berry, R. J. Epigenetic variation in the human cranium. J. Anat., 101(2):361-79, 1967.

Boryslawski, K.; Kurlej, W \& Gworys, B. Changeability of emissary vein foramina based on modern skull material. Dent. Med. Probl., 2:177-82, 2002.

Currarino, G. Normal Variants and Congenital Anomalies in the Region of the Obelion. Am. J. Roentgenol., 127:487-94, 1976.

Gualdi-Russo, E.; Tasca, M. A \& Brasili, P. Scoring of nonmetric cranial traits: a methodological approach. $J$. Anat., 195:543-50, 1999.

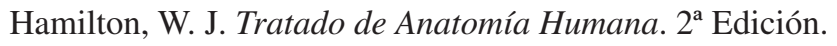
Ed. Interamericana, 1982.

Kutilek, S.; Baxova, A.; Bayer, M.; Leiska, A \& Kozlowski, K. Foramina parietalia permagna: Report of nine cases in one family. J. paediatr. Chid Health., 33:168-70, 1997.

Moyano, B.D.; Estevan, M.; Lizardi, A.S \& Dabezies, A. Agujeros parietales gigantes. A propósito de un caso. Arch. Pediatr Urug., 79(4):303-7, 2008.

Oliva Rodriguez-Pastor, S.; Camacho Alonso, J.M.; Gonzales Gomez, J.M.; Cano España, J \& Calvo Macias, C. Agujeros Parietales. An Esp Pediatr., 55:387-388, 2001.

Reddy, A. T.; Hedlund, G.L \& Percy, A.K. Enlarged parietal foramina. Association with cerebral venous and cortical anomalies. Neurology., 54:1175-8, 2000.

Schaffer, P. J. Morris Human Anatomy. 11ª Ed. New Jork, Mc Graw- Hill Book, 1953.

Tubbs, R. S.; Smyth, M. D \& Oakes, W. J. Parietal Foramina are not Synonymous with Giant Parietal Foramina. Pediatr Neurosurg., 39:216-7, 2003.

Testut, L. \& Latarjet, A. Tratado de Anatomía Humana. 9a ed. Barcelona, Salvat editores, 1971. V.1.
Valente, K. D \& Valente, M. Epilepsy in one family with parietal foramina: an incidental finding?. J. of Neurology Neurosurgery and Psychiatry, 75:1648-9, 2004.

Williams, P. L \& Warwick, R. Gray Anatomia. 35ª ed. Rio de Janeiro, Guanabara Koogan, 1973.

\section{Dirección para correspondencia:}

Prof. Ericka Collipal Larré

facultad de Medicina

Universidad de La Frontera

casilla 54-D

Temuco - CHILE

Email: ecollipa@ufro.cl

Recibido : 22-12-2008 